\title{
The effect of vestibular stimulation on eye-hand coordination and postural control in elite basketball players
} \author{
Hiu Yan Lau", Carina K. Y. Lun", Shamay S. M. Ng ${ }^{1}$ \\ ${ }^{1}$ Department of Rehabilitation Sciences, The Hong Kong Polytechnic University, Hong Kong \\ ${ }^{2}$ Institute of Human Performance, The University of Hong Kong, Hong Kong \\ ${ }^{3}$ Institute of Child Health, University College London, UK
}

William W. N. Tsang ${ }^{1}{ }^{*}$, Shirley S. M. Fong ${ }^{2}$, Yoyo T. Y. Cheng ${ }^{1,3}$, Dinisha D. Daswani ${ }^{1}$,

\section{Email address:}

William.Tsang@polyu.edu.hk (W. W. N. Tsang)

\section{To cite this article:}

William W. N. Tsang, Shirley S. M. Fong, Yoyo T. Y. Cheng, Dinisha D. Daswani, Hiu Yan Lau, Carina K. Y. Lun, Shamay S. M. Ng. The Effect of Vestibular Stimulation on Eye-Hand Coordination and Postural Control in Elite Basketball Players. American Journal of Sports Science. Vol. 2, No. 2, 2014, pp. 17-22. doi: 10.11648/j.ajss.20140202.12

\begin{abstract}
The game of basketball requires complex eye-hand coordination and exceptional postural control ability. This study compared eye-hand coordination and postural control before and after vestibular stimulation in trained basketball players with healthy, age-matched controls. Fifteen trained basketball players and 17 healthy adults (all male, age range 19-25 years) were recruited. The participants were required to perform a fast finger-pointing task involving a moving visual target in a standing position, before and after whole head-and-body rotation at $150^{\circ} \mathrm{s}-1$ for $30 \mathrm{~s}$ seated in a rotational chair. Results show that the trained basketball players had shorter reaction times in eye-hand coordination tasks (a decrease of $23.3 \%$ vs an increase of $8.1 \%$ of controls, $\mathrm{p}=0.008$ ) and regained postural control more quickly (mediolateral direction: $0.4 \%$ vs $43.3 \%$; $\mathrm{p}=0.009$; anteroposterior direction: $3.9 \%$ vs $21.5 \%, \mathrm{p}=0.038$ ) after vestibular stimulation. These data suggest that vestibular stimulation could enhance balance and eye-hand coordination among young basketball players. The findings may provide information for sports training and further research work.
\end{abstract}

Keywords: Eye-Hand Coordination, Postural Control, Vestibular Stimulation, Basketball

\section{Introduction}

Good eye-hand coordination and postural control are essential for superior athletic performances [1-3]. Eye-hand coordination refers to the integrated use of vision, arms, hands and fingers to accomplish goal-directed hand movements [4]. Postural control is the ability to control the centre of mass in relation to the base of support. It depends on the integration of proprioception, vision and vestibular cues in the central nervous system. Among the three sensory cues, vestibular input is particularly important for postural control in sensory challenging environments (e.g. during dynamic sport activities) [5].

Basketball is a dynamic sport where athletes need extremely good eye-hand coordination and postural control for faultless catching, throwing, shooting, jumping, turning and pivoting movements in challenging environments [6].
The complex basketball tasks such as a turn and a jump shot, involve eye-hand coordination combined with excellent postural control. Vestibular input is particularly important for maintaining postural stability during the turning movements [5]. Therefore, it is logical to postulate that vestibular stimulation/strengthening might enhance balance performance and so eye-hand coordination in basketball players. However, to the best of our knowledge, no study has investigated the effect of vestibular stimulation on the aforementioned outcomes thus far.

Based on a study conducted by Tsang and Hui-Chan [7], the horizontal semi-circular canals of the vestibular apparatus can be stimulated by using a rotational chair. This swift rotational movement in the horizontal plane closely simulates the rotational basketball manoeuvres. Therefore, we hypothesize that basketball players might demonstrate better concurrent eye-hand coordination and balance performance after receiving the vestibular stimulation in a rotational chair. 
This study was designed to compare eye-hand coordination and postural control before and after vestibular stimulation in elite basketball players and healthy adults.

\section{Methods}

\subsection{Participants}

Thirty-two males aged 19 to 25 years were recruited by convenience sampling. Fifteen of them were elite basketball players representing their university, and they participated in regular basketball training at least twice per week. The other 17 participants were healthy young active university students who had no regular sport-specific training. Participants who had musculoskeletal injuries such as ankle sprain injury in the previous 6 months or who had a history of vestibular problems or neurological diseases were excluded. All of the participants were screened using a general health questionnaire to ensure that they are healthy individuals. The study was approved by the Human Subjects Ethics Review Committee of the administering University. Written informed consent was obtained from all participants before data collection. All requirements stipulated in the Declaration of Helsinki were conformed.

\subsection{Experimental Set-up}

The equipment used in the experiment included a rotational chair (System 2000; Micromedical Technologies Inc, Chatham, IL, USA), a force platform (Model 9286AA; Kistler, Gommiswald, Switzerland), a visual display unit (VDU) (Clear Tek 3000 LCD screen; MicroTouch Systems Inc., Methuen, USA) and a mono-axial accelerometer (K-beam 8302B10; Kistler, Switzerland). The force platform was placed in front of the rotational chair. The VDU was set at arm's length of each individual standing on the force platform at $90^{\circ}$ of shoulder flexion. The height of the VDU was adjusted to be at each participant's eye level, and the accelerometer was affixed with adhesive tape to the dorsal aspect of the dominant hand at the third meta-carpal phalangeal joint. The equipment was recalibrated before each trial. Each participant performed a standing balance test with concurrent fast finger pointing task toward a moving visual target for three times with the first one as familiarisation trial. The procedure was repeated after vestibular stimulation with a rotational chair. Two minute of rest were given in between each trial of vestibular stimulation.

\subsubsection{Standing Balance with Fast Finger-Pointing}

The participants stood on the force platform with their feet together, arms by their sides for $20 \mathrm{~s}$. Three seconds after stepping onto the force plate, a moving visual target appeared on the VDU. The target was $3 \mathrm{~cm}$ in diameter, and moving at $10 \mathrm{~mm} \mathrm{~s}^{-1}$ from left to right across the screen. The participants were instructed to touch the target with the index finger of their dominant hand as fast and as accurately as possible, and return their hand to their side.

\subsubsection{Vestibular Stimulation}

To stimulate the vestibular system, particularly the horizontal semicircular canals [8], each participant sat in the rotational chair with their head fixed at $30^{\circ}$ of flexion, eyes closed and hands on the armrests. A clockwise whole head-and-body rotation at $150^{\circ} \mathrm{s}^{-1}$ was applied for $30 \mathrm{~s}$. The speed and duration were based on a similar study conducted by Goebel and Paige [9] with young healthy participants aged 20 to 35 years using a rotation speed of $180^{\circ} \mathrm{s}^{-1}$ for $30 \mathrm{~s}$. Here, $150^{\circ} \mathrm{s}^{-1}$ was used because 6 participants reported dizziness and unsteadiness after fast finger-pointing in a pilot study using $180^{\circ} \mathrm{s}^{-1}$. Once the chair had stopped, the participants were instructed to open their eyes, get up as fast as possible, stand on the force platform with their feet together and arms by their sides and perform a finger-pointing task.

\subsubsection{Data Recording}

The age, height, weight, hand dominance and arm length of each participant were recorded. Eye-hand coordination was evaluated in terms of each participant's reaction times, movement times and touch accuracy. Reaction time was defined as the time between the appearance of the visual target on the VDU and the onset of acceleration in the hand movement. Movement time was defined as the time between the onset of acceleration and touching the screen. Touch accuracy was defined as the absolute deviation of the touched location from the centre of the circular target at that moment. The VDU being used in the present study was 34 $\mathrm{cm}$ wide and $27 \mathrm{~cm}$ tall, with resolution of $1024 \times 1024$ and accuracy of $>99 \%$ of true position. The test-retest reliability using such instrumentation has been found to be satisfactory with ICC values range from 0.68 to 0.97 . The known groups validity has also been verified between young and older adults [10].

\subsubsection{Data Reduction}

Postural control was assessed in terms of the time to stabilisation (TTS) in both the mediolateral (ML) and anteroposterior (AP) directions. TTS was used because it can evaluate dynamic postural stability, which is more functional [11]. During the $20 \mathrm{~s}$ standing on the force platform, the trajectory of the centre of pressure (COP) was recorded continuously. The COP data from the force platform were sampled at $100 \mathrm{~Hz}$ and were smoothed using a second-order Butterworth low-pass filter with a cut-off frequency of $0.85 \mathrm{~Hz}$. The sway signals in the ML and AP directions were rectified. The peak values in both the ML and AP directions from 10 to $15 \mathrm{~s}$ and from 15 to $20 \mathrm{~s}$ were detected. For each direction, the lower of the two peak amplitudes was selected for further analysis. The sway signal was then fitted into a polynomial curve with third order, and the amplitude of the curve was checked. The time to stabilisation was defined as the time after which the magnitude of the signal remained smaller than the peak value detected [12,13] (Figure 1). 


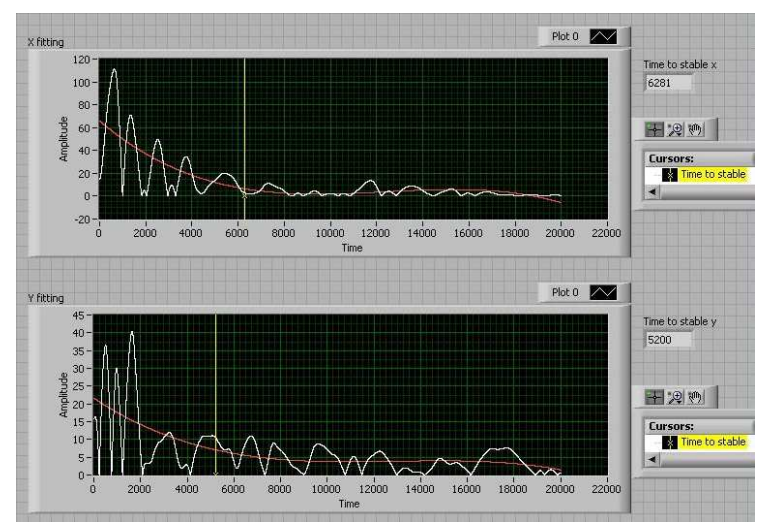

Figure 1. An illustration to demonstrate the determination of the time to stabilization.

\subsubsection{Statistical Analysis}

The average age, weight, height and arm-length of the two groups were compared using independent t-tests. Arm-length was used to normalise the movement time, since a longer arm should require more time to execute a given movement. Paired t-tests with Bonferroni adjustments were performed to compare the eye-hand coordination and time to stabilisation measures before and after vestibular stimulation within each group. Multivariate analysis of variance (MANOVA) was used to compare the eye-hand coordination measures, the times to stabilization, and the percentage changes after vestibular stimulation between the two groups. If statistically significant differences were found, univariate tests were conducted for each outcome measure. The time needed to get up from sitting and step onto the force platform after vestibular stimulation was compared between the two groups using independent t-tests. This was also used as a covariate in the statistical analysis because the effect of vestibular stimulation on balance control could be minimised if participants stayed in the chair too long. Any significant difference in this time interval would then be a confounding variable in between-group comparisons. A significance level $(\alpha)$ of 0.05 (two-tailed) was chosen for statistical comparisons.
All statistical analyses were performed using the SPSS software, version 17.0.

\section{Results}

\subsection{Demographic Data}

Table 1 presents the demographic profiles of the 15 basketball players and the 17 control participants. Independent t-tests showed no statistically significant difference in age between the two groups, but as might be expected, there were statistically significant differences in average height, body weight and arm length between the two groups. Basketball players are generally taller, heavier and have longer arms than other men of similar age.

Table 1. Demographic data of the controls and basketball players.

\begin{tabular}{lccc}
\hline \multirow{2}{*}{ Characteristics } & Health Adults & Basketball players & \multirow{2}{*}{$\boldsymbol{p}$-value } \\
\cline { 2 - 3 } & $(\boldsymbol{n}=\mathbf{1 7})$ & $(\boldsymbol{n}=\mathbf{1 5})$ & \\
\hline Age (year) & $21.1 \pm 0.6$ & $21.3 \pm 1.6$ & 0.643 \\
Height $(\mathrm{cm})$ & $171.2 \pm 4.6$ & $183.2 \pm 7.6$ & $0.003^{*}$ \\
Body weight $(\mathrm{kg})$ & $63.8 \pm 9.7$ & $75.4 \pm 10.2$ & $<0.001^{*}$ \\
Arm length $(\mathrm{cm})$ & $74.5 \pm 3.6$ & $82.1 \pm 5.4$ & $<0.001^{*}$ \\
\hline
\end{tabular}

Note: Values are mean \pm standard deviation for this and subsequent tables.

* Between-group difference significant at $\mathrm{p}<0.05$ (using independent t-tests).

\subsection{Effects of Vestibular Stimulation on Eye-Hand Coordination and Standing Balance Control}

For the healthy young adults, $a$ fter vestibular stimulation, the average reaction time of the control participants increased by $8.1 \%$, movement time decreased by $2.4 \%$ and touch accuracy decreased by $0.8 \%$. However, none of these changes were statistically (or indeed practically) significant (Table 2). The controls did, however, exhibit a highly significant increase in the average time to stabilisation-by $43.3 \%$ in the ML direction and by $21.5 \%$ in AP direction.

Table 2. Comparison of reaction time, movement time, touch accuracy, and time to stabilization before and after vestibular stimulation in healthy controls and basketball players.

\begin{tabular}{|c|c|c|c|c|c|c|}
\hline \multirow{2}{*}{ Measures } & \multicolumn{3}{|c|}{ Healthy Adults ( $n=17)$} & \multicolumn{3}{|c|}{ Basketball Players $(n=15)$} \\
\hline & $\begin{array}{c}\text { Pre-vestibular } \\
\text { Stimulation }\end{array}$ & $\begin{array}{c}\text { Post-vestibular } \\
\text { Stimulation }\end{array}$ & $p$-value & $\begin{array}{c}\text { Pre-vestibular } \\
\text { Stimulation }\end{array}$ & $\begin{array}{c}\text { Post-vestibular } \\
\text { Stimulation }\end{array}$ & $p$-value \\
\hline Reaction time (ms) & $191.4 \pm 49.0$ & $200.8 \pm 58.2$ & 0.539 & $193.3 \pm 66.4$ & $154.5 \pm 33.5$ & 0.117 \\
\hline Movement time (ms) & $770.7 \pm 154.9$ & $737.5 \pm 136.7$ & 0.422 & $706.6 \pm 140.0$ & $791.0 \pm 204.4$ & 0.141 \\
\hline Touch accuracy (mm) & $11.2 \pm 4.5$ & $10.5 \pm 3.9$ & 0.489 & $9.7 \pm 4.6$ & $10.2 \pm 5.2$ & 0.467 \\
\hline \multicolumn{7}{|l|}{ Time to stabilization in: } \\
\hline ML direction (ms) & $6015.9 \pm 1230.4$ & $8266.1 \pm 1113.1$ & $0.000^{*}$ & $5702.0 \pm 1961.1$ & $6589.8 \pm 1831.5$ & 0.297 \\
\hline AP direction (ms) & $6603.7 \pm 1064.2$ & $7850.0 \pm 1237.1$ & $0.004^{*}$ & $6193.5 \pm 1229.1$ & $6856.8 \pm 1400.4$ & 0.121 \\
\hline
\end{tabular}

Notes: $\mathrm{ML}=$ Mediolateral; $\mathrm{AP}=$ Anteroposterior.

* Within-group difference significant at $\mathrm{p}<0.025$ (using paired t-tests; with Bonferroni's adjustment).

For the basketball players, after vestibular stimulation, the average reaction time among the basketball players decreased by $23.3 \%$, movement time increased by $8.8 \%$, and touch accuracy increased by $14.8 \%$. Time to stabilisation in 
ML direction increased by $0.4 \%$, while that in AP direction increased by $4.0 \%$. Paired t-tests confirmed that none of these changes was statistically significant (Table 2).

\subsection{Comparisons of Eye-Hand Coordination and Postural Control between Basketball Players and Healthy Young Adults}

Before vestibular stimulation, the basketball players and the control participants performed similarly with regard to reaction time, movement time, touch accuracy and time to stabilisation in both the ML and AP directions. Multivariate tests of eye-hand coordination results and stabilisation times revealed no significant differences between the two groups.

After Vestibular Stimulation, the time taken to get up from the rotational chair onto the force platform was measured. Basketball players required $2.1 \mathrm{~s}$ on average and the controls required $1.7 \mathrm{~s}$. An independent t-test showed this to be a statistically significant difference between the two groups. Therefore, it was used as a covariate in the multivariate and univariate tests for the analysis of time to stabilisation.

Percentage changes reflecting the differences before and after vestibular stimulation were significantly different between the basketball players and the controls in terms of both the eye-hand coordination measures $(p=0.046)$ and the time to stabilisation $(p=0.008)$. Univariate tests revealed a significantly shorter average reaction time among the basketball players than among the controls (Table 3). However, no significant differences were found in the percentage changes for touch accuracy or movement time. In terms of the time to stabilisation, the controls had a significantly greater percentage increase in both the ML and AP directions when compared with the basketball players.

Table 3. Changes in reaction time, movement time, touch accuracy and time to stabilization for healthy controls and basketball players after vestibular stimulation.

\begin{tabular}{lccc}
\hline & Healthy Adults & Basketball Players & \\
Measures & $(\boldsymbol{n}=\mathbf{1 7})$ & $(\boldsymbol{n}=\mathbf{1 5})$ & $\boldsymbol{p}$-value \\
& percentage change & percentage change & \\
Eye-hand & & & \\
coordination: & & & $0.008^{*}$ \\
$\begin{array}{l}\text { Reaction time (ms) } \\
\text { Movement time } \\
\text { (ms) }\end{array}$ & $-2.4 \pm 18.4$ & $8.8 \pm 22.6$ & 0.137 \\
$\begin{array}{l}\text { Touch accuracy } \\
\text { (mm) }\end{array}$ & $-0.8 \pm 38.3$ & $14.8 \pm 40.3$ & 0.272 \\
$\begin{array}{l}\text { Time to } \\
\text { stabilization in: }\end{array}$ & & & \\
ML direction (ms) & $43.3 \pm 38.3$ & $0.4 \pm 47.1$ & $0.009 *$ \\
AP direction (ms) & $21.5 \pm 25.6$ & $3.9 \pm 24.4$ & $0.038^{*}$ \\
\hline
\end{tabular}

Notes: $\mathrm{ML}=$ Mediolateral; $\mathrm{AP}=$ Anteroposterior.

* Between-group difference significant at $\mathrm{p}<0.05$ using univariate tests, after MANOVA test $\mathrm{p}=0.046$ and $\mathrm{p}=0.008$ for eye-hand coordination measures and time to stabilization, respectively.

\section{Discussion}

The basketball players had a greater average decrease in reaction time after vestibular stimulation. This might be explained in the context of sports psychology by an inverted-U relationship between performance and arousal state. Arousal is a combination of psychological and physiological activity that can be controlled by an individual. A highly aroused person is activated both mentally and physically [14]. The inverted-U hypothesis states that arousal will increase together with performance to an optimal point of best performance. A further increase in arousal will lead to a decrease in performance. A group led by Collardeau [15] has shown that running can improve reaction time during the exercise due to an increase in arousal level. In our study, the vestibular stimulation may have mimicked the swift turning movements in a basketball game. The basketball players may have adapted to the effect of the stimulation and become aroused to their optimal levels for best performance, as in a basketball game. This might have led to the decrease in reaction time after the vestibular stimulation. The controls, on the other hand, may have been over-aroused, leading to slower reaction times. This suggests that basketball trainings may allow the élite basketball players to better regulate their arousal level and focus their attention in performing an eye-hand task in situations like that of a game [14].

A second possible mechanism which might cause shorter reaction times would be "learning effect" due to weekly basketball training. Libet and his co-workers [16] have shown that it takes almost half a second for the brain to be properly conscious of a fast-moving object in the surroundings. In another study, they also demonstrated that it takes 350 to $500 \mathrm{~ms}$ for the mind to complete all the recognition and filtering process to come to a settled field of awareness [17]. In addition, research by Haggard's group [18] suggests that when we anticipate an action to initiate an event, it will appear to happen earlier than if we did not anticipate it. This indicates that consciousness lags reality by approximately half a second, and any more rapid reaction presented by the athletes should have been achieved subconsciously. Basketball involves a lot of speed, turning and eye-hand coordination similar to that set up in these experiments. The elite basketball players may have been able to anticipate the fast-pointing task subconsciously much faster than the controls because they were better accustomed to the effect of vestibular stimulation subconsciously. This would in turn have increased the availability of neural resources for the basketball players to respond to the moving visual target, resulting in the faster reaction times observed.

Under normal circumstances, multisensory information from the vestibular, somatosensory and visual systems is essential to control postural stability. When the accuracy of vestibular input is affected by stimulation, a person uses "sensory channel reweighting" and relies on other sensory inputs, mainly proprioception, to keep the centre of mass (CoM) within his stability limit [19]. If the individual's 
proprioceptive system is not adapted to such demands, such alteration could lead to significant deterioration in postural control. Earlier studies have illustrated the decline in static bipedal standing balance after vestibular stimulation in healthy individuals [20,21].

Previous studies have found that young adults mostly employ ankle torque to minimise AP body sway during stable surface bipedal standing [22]. In such a strategy, synergistic muscle activation sequences are involved. To counteract an anterior sway, the gastrocnemius muscle would first be activated, followed by hamstring and paraspinal muscles. On the other hand, the tibialis anterior, quadriceps and abdominal muscles would be used to resist a posterior sway [5]. When the postural sway amplitude or velocity is too large to be overcome by ankle torque alone, hip torque might also be used in combination with the ankle strategy [23]. Allum and Pfaltz [24] have suggested that coordination of the anterior and posterior muscle systems is maintained through the vestibulo-spinal reflex system. Vestibular input is therefore important in controlling the timing and intensity of the muscle contractions to avoid unidirectional destabilisation. This could explain the delayed AP postural stabilisation observed after vestibular stimulation.

The standardised feet-together stance used in this experiment could have imposed a significant challenge for postural control in the ML direction, leading to the larger percentage change in ML time to stabilisation (43.3\% versus $21.5 \%$ in AP direction) in the control group. With a normal wide stance, ML stability is usually maintained through sideways motion at the pelvis controlled by the hip adductors and abductors. Any stance width narrower than 8 $\mathrm{cm}$ can restrict movement at the hips and induce significant sideways motion at the ankle [25]. However the structural and biomechanical properties of the ankle limit its ML adjustment capacity. Thus, more effortful responses are needed to keep the CoM within the stability limits [26].

The basketball players' better postural control after vestibular stimulation, especially in the ML direction, could be related to their general fitness and sports specific training. Previous studies have shown that percentage lean body mass and muscle strength are positively correlated with postural control [24,27]. Basketball players' ability to generate forces sufficient for controlling their body position in space should also contribute to better postural control [5]. Daily activities such as walking and running involve mostly sagittal plane movement, but basketball training involves foot-work, balance and agility drills performed in multiple directions with lots of turning and pivoting [28]. Such training is designed to impose excessive demands on a player's somatosensory system, muscle recruitment and vestibular system. Adaptations to such training could have led to their superior performance in this experiment.

There are some limitations in this study. First, only male participants between 17 and 20 years old were recruited. Thus, the results may not be generalised to other age groups and to women. Second, the laboratory version of vestibular stimulation was generated by clockwise rotation at a fixed velocity, targeting only on the horizontal semicircular canal. This would certainly be different from the stimulation during dynamic head movement on-court. Postural strategies employed during sports might also be different from that used in bipedal standing. Third, since we used a non-randomized pre-test, post-test control group design in this study, cause-and-effect relationship between vestibular stimulation and balance/eye-hand coordination in basketball players cannot be well established. Further randomised trials are recommended.

Nevertheless, this study serves as a basis for future research into the causal relationship between vestibular stimulation and sports performance. The effect of basketball-related vestibular training for young athletes in general would also be worth exploring.

\section{Conclusions}

The basketball players showed better postural control and faster average reaction times in eye-hand coordination tasks following vestibular stimulation when compared to the control group. On the contrary, young control participants showed a significant increase in time to stabilisation after vestibular stimulation by whole head-and-body rotation. Vestibular stimulation could enhance balance performance and eye-hand coordination among young basketball players.

\section{References}

[1] A.S. Fu, and C.W. Hui-Chan. Ankle joint proprioception and postural control in basketball players with bilateral ankle sprains. Am J Sports Med. 2005, 33, 1174-82.

[2] J. Joyce, J. Graydon, T. McMorris, and K. Devranche. The time course effect of moderate intensity exercise on response execution and response inhibition. Brain Cogn. 2009, 71, 14-9.

[3] H. Nakamoto, and S. Mori. Sport-specific decision-making in a go/ no go reaction task: Difference among nonathletes and baseball and basketball players. Percept Mot Skill. 2008, 106, 163-71.

[4] H. Bekkering, and U. Sailer. Commentary: Coordination of eye and hand in time and space. Prog Brain Res. 2002, 140, 365-73.

[5] A. Shumway-Cook, and M. Woollacott. Motor control: Translating research into clinical practice (3rd ed.). Philadelphia: Lippincott Williams \& Wilkins, 2007.

[6] J.V. Krause, D. Meyer, and J. Meyer. Basketball skills \& drills (3rd ed.). Champaign, IL: Human Kinetics, 2008.

[7] W.W.N. Tsang, and C.W. Hui-Chan. Standing balance after vestibular stimulation in tai chi-practicing and nonpracticing healthy older adults. Arch Phys Med Rehabil. 2006, 87, 546-53.

[8] G.V. Bekesy. Subjective cupulometry: Threshold, adaptation, and sensation intensity of the vestibular organ for rotations in the horizontal plane. AMA Arch Otolaryngol. 1955, 61, 16-28. 
[9] J.A. Goebel, and G.D. Paige. Posturography following rotation: A model of posture control during vestibular dysfunction. Otolaryngol Head Neck Surg. 1990, 102, 722-6.

[10] J.C. Kwok, C.W. Hui-Chan, and W.W. Tsang. Effects of aging and Tai Chi on finger-pointing toward stationary and moving visual targets. Arch Phys Med Rehabil. 2010, 91, 149-55.

[11] M.Y. Shaw, P.A. Gribble, and J.L. Frye. Ankle bracing, fatigue, and time to stabilisation in collegiate volleyball athletes. J Athl Train. 2008, 43, 164-71.

[12] P.A. Gribble, J. Hertel, C.R. Denegar, and W.E. Buckley. The effects of fatigue and chronic ankle instability on dynamic postural control. J Athl Train. 2004, 39, 321-9.

[13] S. Ross, and K. Guskiewicz. Examination of static and dynamic postural stability in individuals with functionally stable and unstable ankles. Clin J Sport Med. 2004, 14, 332-8.

[14] R.S. Weinberg, and D. Gould. Foundations of sport and exercise psychology. Champaign, IL: Human Kinetics, 2007.

[15] M. Collardeau, J. Brisswalter, and M. Audiffren. Effects of a prolonged run on simple reaction time of well-trained runners. Percept Mot Skill. 2001, 93, 679-89.

[16] B. Libet, E. Wright, B. Feinstein, and D. Pearl. Subjective referral of the timing for a conscious sensory experience. Brain. 1979, 102, 193-224.

[17] B. Libet, C. Gleason, E. Wright, and D. Pearl. Time of conscious intention to act in relation to onset of cerebral activity (readiness potential): The unconscious initiation of a freely voluntary act. Brain. 1983, 106, 623-42.

[18] P. Haggard, S. Clark, and J. Kalogeras. Voluntary action and conscious awareness. Nat Neurosci. 2002, 5, 382-5.
[19] R.J. Peterka. Sensorimotor integration in human postural control. J Neurophysiol. 2002, 88, 1097-118.

[20] B.L. Day, A. Severac Cauquil, L. Bartolomei, M.A. Pastor, and I.N. Lyon. Human body-segment tilts induced by galvanic stimulation: A vestibularly driven balance protection mechanism. J Physiol (Lond). 1997, 500, 661-72.

[21] A.E. Pavlik, J.T. Inglis, M. Lauk, L. Oddsson, and J.J. Collins. The effects of stochastic galvanic vestibular stimulation on human postural sway. Exp Brain Res. 1999, 24, 273-80.

[22] F. Horak, and L. Nashner. Central programming of postural movements: Adaptation to altered support surface configurations. J Neurophysiol. 1986, 55, 1369-81.

[23] P. Allard, M.L. Nault, S. Hinse, R. LeBlanc, and H. Labelle. Relationship between morphologic somatotypes and standing posture equilibrium. Ann Hum Bio. 2001, 28(6), 624-33.

[24] J.H.J. Allum, and C.R. Pfaltz. Visual and vestibular contributions to pitch sway stabilisation in the ankle muscles of normals and patients with bilateral peripheral vestibular deficits. Exp Brain Res. 1985, 58, 82-94.

[25] B.L. Day, M.J. Steiger, P.D. Thompson, and C.D. Marsden. Effect of vision and stance width on human body motion when standing: Implications for afferent control of lateral sway. J Physiol. 1993, 469, 479-99.

[26] D.A. Winter. Human balance and posture control during standing and walking. Gait Posture. 1995, 3, 193-214.

[27] L. Angyán, T. Téczely, and Z. Angyán. Factors affecting postural stability of healthy young adults. Acta Physiol Hung. 2007, 94(4), 289-99.

[28] H. Wissel. Basketball: Steps to success (2nd ed.). Champaign: Human Kinetics, 2004. 